Cahiers de recherches médiévales

\title{
La figure de Jules César chez Pétrarque dans les traditions italienne et française des Triomphes
}

\section{Mattia Cavagna}

\section{(2) OpenEdition \\ Journals}

Édition électronique

URL : https://journals.openedition.org/crm/2562

DOI : $10.4000 / \mathrm{crm} .2562$

ISSN : 1955-2424

Éditeur

Honoré Champion

Édition imprimée

Date de publication : 30 juin 2007

Pagination : 73-83

ISSN : 1272-9752

Référence électronique

Mattia Cavagna, «La figure de Jules César chez Pétrarque dans les traditions italienne et française des Triomphes », Cahiers de recherches médiévales [En ligne], 14 spécial | 2007, mis en ligne le 30 juin 2010, consulté le 15 décembre 2022. URL : http://journals.openedition.org/crm/2562 ; DOI : https:// doi.org/10.4000/crm.2562 


\section{ris}

\section{La figure de Jules César chez Pétrarque dans les traditions italienne et française des Triomphes}

Dans un article de 1946, intitulé «Petrarca e Cesare», Guido Martellotti a étudié l'attitude de Pétrarque vis-à-vis de Jules César'. L'analyse de l'œuvre latine du poète, et tout particulièrement de l'Afrique et du De viris illustribus, lui a permis de montrer que cette attitude évolue vers une réévaluation de ce personnage.

L'Afrique, rédigée en grande partie entre 1337 et 1343, est considérée comme le premier ouvrage de Pétrarque. Il s'agit d'un poème épique-historique en hexamètres latins, dédié à Robert d'Anjou, roi de Naples (mort en 1343). Scipion l'Africain est le protagoniste de l'ouvrage; la narration porte sur les guerres puniques, avec des effets d'annonce et de prophéties concernant la gloire future de Rome. Le De viris illustribus, commencé en 1338, est un recueil de biographies des personnages de l'Antiquité romaine. Selon le projet originaire, il aurait du comprendre vingt-trois biographies des hommes illustres depuis Romulus jusqu'à Titus, avec quelques personnages qui se situent en dehors de Rome (Alexandre, Pyrrhus, Hannibal), mais la première rédaction s'arrêta à la biographie de Néron. Par la suite, entre 1351 et 1353 , Pétrarque rajouta douze biographies de personnages de l'Ancien Testament et de figures mythiques de la Grèce, notamment Jason et Hercule, mais la biographie de ce dernier resta inachevée.

L'Afrique témoigne du fait que, dans sa jeunesse, Pétrarque avait élu Scipion comme le principal héros de l'Antiquité. Dans le dernier livre du poème, l'auteur annonce son projet du De viris illustribus, en précisant que la vie de Scipion sera la plus grande et la plus belle: «Au milieu de ce même ouvrage, éclatant et formant une part importante de l'œuvre, on trouvera le grand Scipion $»^{2}$. Le personnage de César, par contre, est jugé avec sévérité. Dans le $\mathrm{II}^{\mathrm{e}}$ livre, inspirée du Songe de Scipion de Cicéron, le père de Scipion présente un excursus des futures gloires de Rome. César est célébré d'abord pour ses victoires contre les Gaules et les Germains, mais juste après il est condamné pour avoir levé les armes contre sa propre patrie. «Dans le trouble de son esprit, il retournera ses mains partout victorieuses contre le cœur de la république, souillant des batailles étrangères par le sang de ses concitoyens et ses triomphes légitimes par des guerres indignes $»^{3}$.

\footnotetext{
${ }^{1}$ G. Martellotti, «Petrarca e Cesare », Annali della Scuola Normale Superiore di Pisa, XVI, (1947), pp. 149-158, rééd. dans : Scritti Petrarcheschi, Padoue, Antenore, 1983, p. 77-89.

${ }^{2}$ In medio effulgens nec corpore parvus eodem / Magnus erit Scipio (Africa, IX, 261). Nous citons le texte d'après l'éd. et la traduction française de R. Lenoir, Pétrarque, L'Afrique, Grenoble, Million, 2002 (ici, p. 432-433).

${ }^{3} \mathrm{O}$ felix si forte modum sciat addere ferro!

Nesciet heu! noletque miser; sed turbine mentis

Victrices per cunta manus in publica vertet

Viscera, civili fedans externa cruore

Prelia (Africa II, v. 228-231). Ibid., p. 90-91.
}

Cahiers de Recherches Médiévales, 14spé, 2007 
Dans ce même ouvrage, Pétrarque attribue à César une responsabilité lourde : celle d'avoir proposé le premier exemple d'un pouvoir absolu et d'avoir profité du Trésor Public au début des guerres civiles. Dans cette œuvre de jeunesse les deux héros, Scipion et César se trouvent donc, en quelque sorte, en opposition.

Pétrarque est très loin du culte et de l'admiration pour l'empire, typique de la production de l'Italie médiévale. À ce propos, il suffira de rappeler que dans la $D i$ vine Comédie Brutus et Cassius, les meurtriers et traîtres de César, sont explicitement assimilés à Judas, le traître de Jésus Christ: les trois sont placés au fond de l'enfer, dans les trois gueules de Lucifer. Dante partage avec ses contemporains une admiration sans bornes pour Jules César qui est considéré comme le fondateur de l'empire, là où l'empire est considéré, à son tour, comme la réalisation sur terre de l'ordre divin. Aux yeux de Pétrarque, par contre, César incarne tous les dangers du pouvoir absolu et tyrannique qui s'oppose aux idéaux républicains.

Et pourtant, au cours des années, l'attitude de Pétrarque change assez radicalement, si bien que son admiration pour César dépassera même celle pour Scipion, comme en témoigne la lecture des deux biographies contenues dans le De viris illustribus. Contrairement au projet qu'il avait annoncé dans l'Afrique, la biographie de Jules César est en effet de loin la plus développée: dans les dernières années de sa vie, Pétrarque l'amplifie énormément et lui confère un statut autonome en lui attribuant le titre De gestis Cesaris'.

Une telle évolution est liée à l'approfondissement de la culture du poète, notamment à une analyse plus minutieuse de la biographie de Suétone, à la découverte, en 1345, des épîtres Ad Atticum de Cicéron et surtout à la lecture des Commentarii, qui exercèrent sur Pétrarque une grande fascination, en lui permettant de suivre le héros dans la dimension la plus intime de sa vie ${ }^{5}$. L'admiration qui se développe chez Pétrarque porte moins sur le rôle symbolique du héros, lié à l'instauration de l'empire, que sur sa personnalité et sur ses qualités humaines. Parmi les anecdotes les plus célèbres qui frappèrent Pétrarque, nous nous limiterons à signaler l'épisode des larmes versées par César sur le corps de son ennemi Pompée, anecdote relaté par Suétone (De viris illustribus, II, 544).

Au lieu de revenir sur l'œuvre latine de Pétrarque, qui a déjà été largement analysée en ce sens par Martellotti et par bien d'autres critiques ${ }^{6}$, nous aimerions concentrer notre attention sur un ouvrage italien, les Triomphes, à l'intérieur duquel Jules César occupe également un rôle de tout premier plan. Nous proposerons une

\footnotetext{
${ }^{4}$ La Bibliothèque Nationale de France conserve le manuscrit autographe de cet ouvrage, sous la cote lat. 5784.

${ }^{5}$ Probablement Pétrarque ignorait que l'auteur des Commentarii est César lui-même, cf., à ce propos, U. Bosco, «Il Petrarca e l'umanesimo filologico », Giornale storico della letteratura italiana, 120 (1942), rééd. dans Saggi sul Rinascimento italiano, Florence, Le Monnier, 1970, p. 186-187.

${ }^{6}$ Cf., par exemple, l'article récent de D. Ménager, «La Figure de César dans les recueils biographiques de la Renaissance », Cahiers de Recherches Médiévales, 13spécial (2006), La Figure de Jules César au Moyen Âge et à la Renaissance, p. 9-21
} 
analyse portant sur le texte original de Pétrarque et sur sa tradition italienne et française, constituée d'une série de commentaires et de traductions.

La rédaction des Triomphes se situe autour des années 1340-134. L'ouvrage est resté inachevé et n'a été publié qu'après la mort de l'auteur ${ }^{7}$; il est composé d'une série de six cortèges allégoriques, formées par de très nombreux personnages célèbres, placés sous l'égide d'une figure allégorique. Les six parties sont pourvues de titres latins: Triumphus Cupidinis, Triumphus Pudicitie, Triumphus Mortis, Triumphus Fame, Triumphus Temporis, Triumphus Eternitatis (Triomphe de l'Amour, de la Chasteté, de la Mort, de la Renommée, du Temps, de l'Éternité); elles sont constituées d'un nombre inégal de sous-parties, pour un total de quatorze chapitres. La figure de Jules César se trouve placée à deux endroits stratégiques, à savoir dans le Triomphe de l'Amour et dans le Triomphe de la Renommée : dans les deux cas, il est la première des très nombreuses figures allégoriques citées.

Le Triomphe de l'Amour présente tous les personnages dont la célébrité est liée justement à leur propension pour l'amour charnel. César ouvre ce cortège et est donc le premier personnage historique nommé dans les Triomphes.

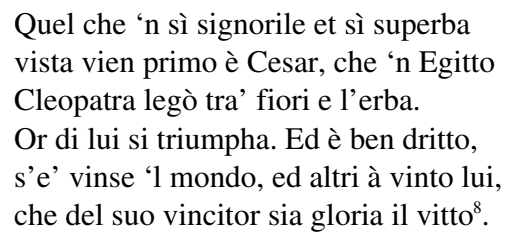

César bénéficie de cette place d'honneur dans le cortège à cause de son amour pour Cléopâtre, qui parvint à le séduire et à se faire proclamer reine d'Egypte. Selon la logique des triomphes, l'importance de la victoire remportée par Amour est proportionnelle à la puissance du vaincu ; c'est pourquoi César, qui a triomphé du monde entier et qui a été vaincu par Amour, mérite la première place dans le cortège. César est suivi par une longue série de personnages appartenant à l'histoire et à la mythologie (Octavien Auguste, Néron, Marc Aurèle, Enée, Alexandre, Achille etc.) et par une série de couples célèbres (Jason et Médée, Mars et Venus etc.).

Le Triomphe de la Renommée présente la série des personnages qui se sont distinguées par leurs vertus guerrières. Une fois encore, César ouvre le cortège, mais cette fois-ci sa prééminence est, pour ainsi dire, remise en question d'abord par Pétrarque lui-même et, ensuite, par les commentateurs, italiens et français, qui se sont penchés sur ce passage. Tout d'abord, on notera que ce Triomphe nous a été

\footnotetext{
${ }^{7}$ Nous lisons le texte dans l'édition de V. Pacca, Triumphi, dans Francesco Petrarca. Trionfi, Rime estravaganti, Codice degli abbozzi, éd. V. Pacca et L. Paolino, introduction de M. Santagata, Milan, Mondadori, 1996.

${ }^{8}$ Triumphus Cupidinis I, v. 88-93. Ibid., p. 74. "Celui qui avance le premier, avec une apparence si noble et si superbe, est César, que Cléopâtre en Egypte enserra dans des liens de fleurs et de verdure. Or l'on triomphe de lui, et il est bien juste, puisqu'il vainquit le monde et qu'un autre l'a vaincu, que le vaincu soit la gloire du vainqueur». (À partir de ce passage, toutes les traductions des citations italiennes sont personnelles).
} 
transmis en deux rédactions différentes. Dans la première rédaction, César se trouve tout seul à la droite de la déesse de la Renommée :

Ella a veder parea cosa divina,

e da man destra avea qual gran romano

che fe' in Germania e 'n Francia tal ruina'.

On notera que le nom de César n'apparaît pas et qu'il est désigné à travers la dénomination «grand romain». César est suivi d'Octavien Auguste et de Claude Néron Druse, fils adoptif de ce dernier, célèbre pour ses batailles contre les Germains. Scipion l'Africain n'occupe que la quatrième place.

Dans la rédaction définitive pourtant, Scipion revient à l'honneur et rejoint César à la tête du cortège :

Da man destra, ove gli occhi in prima porsi,

la bella donna avea Cesare e Scipio,

ma qual più presso a gran pena m' accorsi ;

l'un di vertute, et non d'Amor mancipio,

l'altro d'entrambi ${ }^{10}$.

L'antinomie César - Scipion, qui caractérise le premier ouvrage de Pétrarque est mise ici définitivement de côté. Les deux personnages rentrent dans le texte au même titre si bien que, dans le char de la Renommée, le poète n'arrive pas à établir qui des deux se tient plus prés de la personnification allégorique. Le seul élément qui distingue les deux personnages concerne leur rapport avec l'amour : l'un (César) est assujetti à la vertu et à l'amour alors que l'autre (Scipion) seulement à la vertu.

Dans la riche tradition des commentaires et des traductions des Triomphes, ce passage du Triomphe de la Renommée occupe une place centrale car il a fait l'objet de plusieurs longues gloses et de plusieurs interprétations divergentes que nous analyserons dans la présente étude ${ }^{11}$.

En Italie, il existe trois commentaires de cet ouvrage qui datent $\mathrm{du} \mathrm{XV}^{\mathrm{e}}$ siècle. Le premier est de Bernardo Ilicino, savant médecin de Sienne; le deuxième est

\footnotetext{
${ }^{9}$ Triumphus Fame Ia, v. 22-24. Ibid., p. 558. «À la voir, elle paraissait chose divine, et à sa droite elle avait ce grand Romain qui fit en Allemagne et en France une telle ruine ».

${ }^{10}$ Triumphus Fame I, v. 22-26. Ibid., p. 356. «À sa droite, où je posai d'abord mes yeux, la belle dame avait César et Scipion, mais je ne pouvais déterminer le plus proche ; l'un assujetti à Vertu et non à l'Amour, l'autre aux deux ».

${ }^{11}$ Mis à part les commentaires des Triomphes, il faut rappeler que la question de la supériorité de César ou de Scipion fait l'objet d'un véritable débat dans le milieu humaniste italien du $\mathrm{XV}^{\mathrm{e}}$ siècle. Les protagonistes principaux de ce débat sont Poggio Bracciolini et Guarino Guarini : le premier célèbre la république de Florence, où le seigneur De Médicis pouvait être comparé à Scipion, le deuxième célèbre la principauté de Ferrare, et notamment les ducs d'Este, à travers la figure de Jules César. Cf., à ce propos., l'étude de G. Crevatin, «La politica e la retorica. Poggio e la controversia fra Cesare e Scipione», dans Poggio Bracciolini (1380-1980), Florence, Sansoni, 1982, p. 281-342.
} 
de Jacopo di Poggio Bracciolini, florentin, fils du célèbre Poggio Bracciolini; le troisième a été attribué, à la fin du $\mathrm{XV}^{\mathrm{e}}$ siècle, à l'humaniste Francesco Filelfo, mais son auteur reste inconnu. D'autre part, il existe deux traductions françaises : la première, en vers, reprend le texte de manière littérale ; la deuxième, en prose, intègre la tradition des commentaires et s'appuie en large partie sur le commentaire de Bernardo Ilicino même si, comme on le verra, l'approche à la matière et la nature du commentaire sont très différentes par rapport à la tradition humaniste italienne ${ }^{12}$.

\section{Le commentaire de Bernado Ilicino}

Composé entre 1468 et 1469 , ce commentaire a eu une diffusion très large, quoique de brève durée, comme en témoignent les quelques vingt-trois différentes éditions imprimées, réalisées entre 1475 et $1522^{13}$. Il a été également traduit en français ${ }^{14}$.

Bernardo Ilicino propose une glose au texte en développant la présentation de chacun des personnages que Pétrarque fait défiler dans les cortèges allégoriques. Au niveau de la mise en page, l'œuvre présente les caractéristiques typiques de la glose médiévale : le texte, imprimé en grands caractères, est confiné sur la marge gauche et est entouré de son commentaire, imprimé en caractères beaucoup plus réduits. En revanche, le contenu de la glose s'écarte fortement de la tradition des commentaires moraux de la tradition médiévale : loin de poursuivre un but édifiant ou pédagogique, en soulignant la portée exemplaire de tel ou tel personnage, le commentateur se propose avant tout de comprendre et de justifier les choix opérés par Pétrarque dans la construction de son ouvrage ${ }^{15}$.

Dans le commentaire sur Jules César et Scipion, Ilicino considère tout d'abord le rapport entre la renommée d'armes et celle des lettres, un thème qui a suscité des débats importants dans le monde humaniste. Ensuite, il présente des brèves - relativement brèves - notices biographiques des deux personnages, en sélectionnant une série de gestes témoignans de leurs vertus. Finalement, il revient sur la question de la parité entre les deux personnages, en s'efforçant d'expliquer le choix de Pétrarque :

Non senza ragione il nostro M. Francesco dice essere difficile a iudicare chi di questi due exceda in fama et sia superiore, imperciò che se Cesaro vinse più populi Sci-

\footnotetext{
${ }^{12}$ La traduction en vers est en cours d'édition par les soins de G. Parussa et E. Suomela Härmä, La première traduction en vers des Triomphes de Pétrarque par Simon Bourgouin (à paraître). Nous remercions chaleureusement Gabriella Parussa pour ses précieuses suggestions et pour son soutien amical.

${ }^{13}$ Nous lisons le commentaire dans l'édition imprimée à Venise, en 1515, dans l'exemplaire conservé à BNF, sous la cote Rés. YD 79.

${ }^{14}$ La traduction est conservée dans le manuscrit de Paris, BNF fr. 594.

15 À propos de ce commentaire, voir surtout S. Cracolici, «Esemplarità ed emblematica nel commento di Bernardo Ilicino ai Triumphi di Petrarca», dans I Triumphi di Francesco Petrarca, Acte du Colloque de Gargnano del Garda (1-3 oct. 1998) éd. C. Berra, Milan, Istituto Editoriale Universitario, 1999, p. 403-417 (ici, p. 411). Nous tenons à remercier Stefano Cracolici pour les conseils précieux qu'il nous a transmis au moment de la préparation de la présente étude.
} 
pion ne domò più feroci ; maximamente, secundo che scrive Vegetio in Liber de re militari, se Cesaro acquistò maggiore dominio alla romana repubblica, Scipione la liberò da più grave periculo; se Cesaro fu liberale Scipione clementissimo; se Cesaro fu clarissimo oratore Scipione fu insegne ; se Cesaro dominò la romana repubblica Scipione signoreggiò li Africani, essendo universalmente dal populo eletto consule nella guerra africana; etiamdio contrariante il senato ${ }^{16}$.

Son effort d'argumentation le porte à vouloir effacer même la petite différence que Pétrarque avait mise en évidence dans le rapport des deux avec l'amour :

Se a Cesaro se attribuisce più leggiadria per lo esser stato inamorato, a Scipione si dà più continentia per lo havere a tal amore repugnato ${ }^{17}$.

Dans sa comparaison systématique entre les deux héros, Bernardo Ilicino force le texte de Pétrarque jusqu'à interpréter la passion amoureuse de César comme une vertu - la "grâce»(leggiadria) - qui peut être mise sur le même plan que la « chasteté » (continentia) de Scipion ${ }^{18}$.

Ilicino veut à tout prix niveler, pour ainsi dire, tous les mérites des deux personnages, afin de comprendre et de justifier le choix de Pétrarque. Comme l'explique Stefano Cracolici, les deux notices biographiques, d'ampleur identique, qui présentent les gestes des deux héros, vont également dans ce sens : elles offrent au lecteur la possibilité de vérifier lui-même la difficulté objective d'établir qui, parmi César et Scipion, est le plus digne d'éloges ${ }^{19}$. Le même Stefano Cracolici insiste sur l'altérité de ce commentaire par rapport à la tradition exégétique médiévale: d'après son interprétation, Ilicino se montre parfaitement conscient qu'il se trouve devant un répertoire nouveau de personnages illustres, présentés dans un ordre hiérarchique précis. En laissant de côté tout jugement moral, il s'efforce de comprendre et d'expliquer la logique interne à la construction de ce répertoire. Du moment où les personnages sont inscrits dans un Triomphe, du moment où le commentaire justifie cette position, leur portée exemplaire, passe au deuxième plan par rapport à leur valeur triomphale, à la place qu'ils occupent maintenant, en tant

\footnotetext{
${ }^{16}$ Paris, BNF, Rés. YD 79, fol. 66v. «Ce n'est pas sans raison si notre M. Francesco dit qu'il est difficile de juger lequel de ces deux personnages est supérieur en renommée, puisque si César vainquit plus de peuples, Scipion en vainquit de plus féroces; d'après ce qu'écrit Végèce dans le Liber de re militari, si César acquit un territoire plus grand à la république romaine, Scipion la délivra d'un péril plus grand; si César fut libéral, Scipion fut très clément; si César fut un grand orateur, Scipion fut insigne ; si César domina la république romaine, Scipion imposa son pouvoir sur les Africains, ayant été élu par l'unanimité du peuple comme consul de la guerre africaine, même contre l'opinion du sénat ».

${ }^{17}$ «Si à César on attribue plus de grâce pour avoir été amoureux, à Scipion on attribue plus de continence pour avoir répudié l'amour ».

${ }^{18}$ La traduction française du commentaire a recours à des couplets synonymiques : Se a Cesar est attribué plus de plaisir et humanité pour avoir esté amoureux, a Scipion est attribué plus de louenge par sa continence pour avoir a tel amour repugné et resisté (ms BNF, fr. 594, fol. 204v).

${ }^{19}$ S. Cracolici, art. cit., p. 412-413.
} 
qu'emblèmes, à côté de la renommée. Dans les Triomphes, Pétrarque se fait témoin et acteur d'une évolution importante : celle qui porte de la tradition de l'exemplum à celle de l'emblème. Si le personnage exemplaire est situé dans un contexte narratif et se prête à une interprétation morale, le personnage emblématique est complètement soustrait de la narration et s'impose comme l'incarnation même d'une vertu ${ }^{20}$.

\section{Le commentaire du pseudo-Filelfo}

Ce commentaire a été imprimé à Parme, en 1473, par l'éditeur Andrea Portilia et est connu sous l'appellation de chiose Portilia ${ }^{21}$. En lisant le commentaire du passage en question, on comprend bien que l'auteur poursuit un but très proche de celui d'Ilicino, à savoir celui de supporter le choix de Pétrarque, en confirmant la parité des deux personnages. Et pourtant, l'auteur anonyme atteint ce même but en parcourant une voie qui est, en quelque sorte, antithétique par rapport à celle de son prédécesseur: au lieu de réinterpréter la luxure de César en la considérant comme une vertu, il attribue à Scipion un péché de luxure.

Egli, poi che tributaria Cartagine al popolo di Roma forse per intermissione delle passate fatiche amò la sua serva et con licentia di Livia sua moglie si posoe con essa. Et come ogni huomo savio deve extimare et credere, lui forse come huomo non poté manchare che non errasse nel mondo alcuna volta. Adunche, poi che triomphoe di Syface, re di Numidia et Mauritania, perfino agli monti di Varco verso l'oceano et della superba Caragine, Amore triomphoe di lui reducendolo con alcuna humanitate ad suo volere ${ }^{22}$.

Ce commentateur s'efforce de jeter une ombre sur Scipion, en montrant que lui aussi, au fond, a été la victime de la passion amoureuse, une passion qu'il tend à identifier d'un côté avec la «faiblesse » et, de l'autre, avec l' «humanité ». Il précise entre-temps que la faute de Scipion n'est pas très grave, puisqu'il a obtenu le consentement de sa femme. Cet épisode est basé sur une source historique importante : Valère Maxime, Facta ac dicta memorabilia $(67 \text { 1-3 })^{23}$.

\footnotetext{
${ }^{20}$ Le caractère statique de la tradition des emblèmes contribue à expliquer son succès dans le domaine de l'iconographie. Cf. L. Battaglia Ricci, «Immaginario trionfale: Petrarca e la tradizione figurativa », dans I Triumphi di Francesco Petrarca, op. cit., p. 259-298.

${ }^{21}$ À propos de ce commentare, cf. l'étude de F. Tateo, «Sulla ricezione umanistica dei Trionfi », dans I Triumphi di Francesco Petrarca, op. cit., p. 375-401.

${ }^{22}$ Nous citons le texte du manuscrit de Berlin (78 D 11 fol. 183-183v) d'après la transcription de F. Tateo, Ibid. p. 391. «Ce dernier (Scipion), après avoir assujetti Carthage au peuple de Romme, peut-être pour se remettre de sa peine, aima sa servante et, avec la permission de Livie sa femme, coucha avec elle. Et, selon ce que tous les hommes sages doivent croire, lui, en tant qu'homme, il ne put pas s'empêcher de commettre quelques fautes au cours de sa vie. Après qu'il triompha de Syphax, roi de Numidie et de Mauritanie, jusqu'aux montagnes de Varco, vers l'océan, et de la superbe Carthage, Amour triompha de lui en le réduisant avec humanité à son vouloir».

23 Dans la source latine, Valère Maxime, l'épisode a probablément une signification allégorique: la servante, amante de Scipion, symbolise vraisamblablement la ville de Carthage, qui a été justement asujettie.
} 


\section{Le commentaire de Jacopo di Poggio Bracciolini}

Ce commentaire, dédié à Lorenzo il Magnifico, a été publié à une date comprise entre 1475 (date de la première édition du commentaire d'llicino) et 1478 (mort de l'auteur) ${ }^{24}$. L'attitude de l'auteur se distingue assez nettement par rapport à celle de ses prédécesseurs, dans la mesure où il montre une forte hostilité vers la figure de Jules César. Et pourtant, malgré cette orientation fortement polarisée, l'effort de l'auteur est toujours concentré sur la compréhension du choix de Pétrarque.

Dans le passage qui suit, Jacopo Bracciolini mentionne les deux versions du Triomphe de la Renommée en les considérant comme deux chapitres successifs du même texte, mais il les situe dans un ordre inversé, si bien que la première version, celle où César se trouve tout seul à côté de la déesse, est considérée comme la version définitive ou, en tout cas, successive à la deuxième :

Onde è da maravigliare, havendo in qualunque sua opera preposto Scipione superiore non solo a Cesare, ma a ciascuno altro, e in un altro capitulo di questo medesimo triompho non haver voluto dar sententia chi dovessi precedere, in questo luoco solo facci tanta mutatione d'animo : perché veramente è da credere che lui preponesse Cesare non come huomo più virtuoso, ma più famoso. $\mathrm{E}$ benché secondo el giudicio de' savii Scipione meriti maggior laude, niente dimeno Cesare nella bocca del vulgo è più nominato, et essendo più nominato ha magior fama. E nel capitulo della fama non chi più merita, ma chi è in più fama debba essere preposto ${ }^{25}$.

Le commentateur exprime, tout d'abord, son étonnement vis-à-vis de l'attitude de Pétrarque et de son soudain revirement. Il rappelle que, dans tous ses ouvrages, le poète a toujours célébré Scipion en le considérant comme le plus grand héros de l'Antiquité. Puis, dans un autre chapitre des Triomphes, c'est-à-dire dans la deuxième version du Triomphe de la Renommée, il a placé Scipion sur le même plan que César et finalement, dans le passage qu'il a sous les yeux - c'est-à-dire dans la première version du Triomphe de la Renommée - il a complètement changé d'avis en conférant à César la priorité absolue ce qui, visiblement, heurte fortement la sensibilité du commentateur.

Cependant, loin de remettre en discussion l'auctoritas de Pétrarque, Jacopo Bracciolini fournit une explication de caractère philosophique: la renommée ne coïncide guère avec la vertu et César, loin d'être célébré par sa vertu, se trouve dans

\footnotetext{
${ }^{24} \mathrm{Ce}$ commentaire a été également analysé par F. Tateo, Ibid.

${ }^{25}$ Ibid., p. 389. «C'est donc bien étonnant que, après avoir placé Scipion au-dessus non seulement de César, mais aussi de tous les autres, dans tous ses ouvrages, et après avoir refusé, dans un autre chapitre de ce même triomphe, de déterminer qui d'entre eux mérite de précéder l'autre, il (Pétrarque) change d'avis si radicalement. Car il faut vraiment croire qu'il donne la priorité à César non pas en tant qu'homme vertueux, mais en tant qu'homme célèbre. Et malgré le fait que, selon le jugement des sages, Scipion mérite plus d'éloges, César, dans la bouche du peuple, est nommé bien plus souvent et en ce sens il a plus de renommée. Au chapitre de la Renommée on accorde la priorité non pas à celui qui a le plus de mérite, mais à celui qui bénéficie d'une plus vaste renommée».
} 
une position de priorité puisque son nom est «dans la bouche du peuple» (le terme vulgo a, bien sûr, une connotation dépréciative). Ce commentaire déplace donc l'enjeu de la question en attirant l'attention sur la signification ambiguë de la renommée, sur son caractère éphémère, vain et souvent immérité.

Le rapport César - Scipion et les raisons du choix de Pétrarque sont toujours au centre du débat mais à la différence des autres commentateurs, Jacopo Bracciolini prend position en faveur de l'un des deux héros et dans la suite de son commentaire émet un jugement très sévère sur César. Il tranche d'une manière très nette entre les qualités de Scipion et les vices de César et arrive même à opposer la «renommée immortelle » (fama immortale) de Scipion et «l'infamie éternelle (infamia sempiterna) de César, ce qui - de toute évidence - n'est pas vraiment en syntonie avec le discours de Pétrarque ${ }^{26}$.

\section{La traduction française en prose}

Venons-en finalement à la traduction française en prose, qui a été imprimée au moins cinq fois entre 1514 et 1539 et qui a été attribuée à tort à Georges de La Forge. La première édition, qui constitue notre édition de référence, a été réalisée à Paris, dans l'atelier de Barthélemy Vérard, en $1514^{27}$. Cette traduction réunit dans le corps du texte la mise en prose des vers de Pétrarque et la tradition du commentaire, sans établir un rapport dialectique entre le texte et sa glose. Le commentaire est original seulement en partie: l'auteur propose souvent sa propre exégèse du texte mais a également recours à d'autres commentaires, et tout premièrement à celui de Bernardo Ilicino, qu'il traduit de l'italien. Les autres sources restent à identifier.

Par rapport à la tradition du texte et des commentaires, la structure de l'ouvrage subit des modifications assez radicales. L'auteur choisit une série de figures insérées dans les cortèges et développe leurs vies, en leur consacrant des portions de texte assez inégales. Il insère des titres des chapitres, mais de façon très irrégulière. Le texte est également accompagné de gravures qui sont placées, le plus souvent, à côté des rubriques des chapitres.

Le passage qui nous intéresse, et qui met en scène César et Scipion à côté de la Renommée, fait justement l'objet d'un chapitre dont le titre et la présentation sont assez significatifs. Le titre, DE JULIUS CESAR EMPERIERE, est suivi par une grande gravure représentant Jules César sur un trône, avec son épée. Un personnage agenouillé à son chevet lui offre une sphère, symbole du pouvoir. D'après ces éléments du para-texte, Jules César semble bénéficier ici d'une prééminence absolue, si bien que son «concurrent»n'est même pas mentionné. La traduction du passage, pourtant, suit le texte de Pétrarque d'une manière très fidèle :

\footnotetext{
${ }^{26}$ Per la qual cosa, sendo la virtù sola quella che facci degni di loda e fama immortale gli uomini prestanti e vicii detestandi e d'infamia sempiterna, non è dubio Scipione per la virtu sua haver grandemente superato Cesare, nell'arte militare e populi e capitani subjugati in niuna cosa inferiore. Liberalità beneficentia, pudicizia, castità di Scipione; di Cesare furti, rapine, lascivie assai si dimostrano. Ibid .

${ }^{27}$ Voici une liste des autres réimpressions du texte : Paris, Jehan de La Garde, 1519 ; Paris, Hémon Le Fèvre, 1520 ; Lyon, Denys de Hersy, 1531 ; Paris, Denys Janot, 1539.
} 


\begin{abstract}
Adonc, ainsi que retournoye mes yeulx a la main dextre du chariot triumphal d'icelle dame renommee, je vy que auprés de soy elle avoit triumphans deux tresvertueux hommes, c'est assavoir Cesar et Scipion, mais il estoit difficile a juger lequel des deux estoit d'elle plus favorite. Touteffois, ilz estoient excellemment fameux, mais diversement renommez, car l'ung estoit serf et mancipe de vertu et non point d'amour, et l'autre militoit entre deux, c'est assavoir entre amour et vertu ${ }^{28}$.
\end{abstract}

La dernière sentence, qui souligne la différence ente les deux héros, fait l'objet du commentaire suivant :

Scipion, suyvant vertu, tenoit et combatoit pour l'amour d'autruy, c'est assavoir pour le bien commun et chose publicque de Romme, mais Cesar, tout au contraire, avoit la vertu par laquelle il tenoit et combatoit pour l'amour de soy, c'est assavoir quant il applicqua et conquist a soy particulierement contre la chose publicque de Romme l'empire rommain ${ }^{29}$.

S'ensuivent les biographies des deux, tirées directement des commentaires de Bernardo Ilicino. Ce bref passage montre que l'attitude de ce commentateur se distingue assez nettement par rapport à celle de ses «collègues» italiens. Le commentateur français soumet le texte de Pétrarque à une interprétation de type moral. L'amour, qui dans le texte original renvoie, bien évidemment, à l'amour érotique, est interprété ici comme «amour de soy », c'est-à-dire comme un péché d'orgueil, ce même péché qui a porté César à se révolter contre sa propre patrie et contre «la chose publicque de Romme». Dans sa glose, l'auteur revient ici sur le reproche qui avait été formulé par Pétrarque lui-même dans l'Afrique. Son commentaire est bel et bien une glose morale et s'inscrit mal dans la tradition des commentaires humanistes $\mathrm{du} \mathrm{XV}^{\mathrm{e}}$ siècle, en restant ancré à un système d'exégèse strictement médiéval.

Il est intéressant de souligner le décalage qui existe entre les éléments du paratexte, entièrement focalisés sur la figure de Jules César en majesté, et le commentaire, qui par contre adresse tous ses éloges à Scipion et soumet César à un jugement sévère. Un tel décalage tient sans doute à l'organisation interne de l'imprimé, où la composition du para-texte, et tout particulièrement le choix et la distribution des illustrations, repose traditionnellement sur l'éditeur et relève d'une opération commerciale. C'est pourquoi, d'après notre interprétation, Barthélemy Vérard a cru opportun attirer l'attention du public sur le personnage de César, un personnage bien plus «vendable», par rapport à Scipion, en raison - justement - de sa renommée universelle.

\title{
Conclusion
}

Cet excursus de textes nos a permis de montrer, avant tout, que la figure de Jules César constitue un centre d'intérêt majeur dans le milieu humaniste du $\mathrm{XV}^{\mathrm{e}}$ siècle. Plusieurs des commentateurs de Pétrarque ont focalisé leur attention sur le passage qui présente l'empereur à côté de Scipion, en offrant des lectures, des analyses et des interprétations divergentes, mais surtout en abordant la question sous des

${ }^{28}$ Nous lisons le texte dans l'exemplaire conservé à Paris, BNF, Rés. YD 80, fol. 65v-66r.

${ }^{29}$ Ibid. 
approches herméneutiques assez différentes. Bernardo Ilicino a recours à son habilité rhétorique en réinterprétant la passion amoureuse de César comme une sorte de vertu (la «grâce ») et en la mettant sur le même plan que la chasteté de Scipion. Le pseudo-Filelfo a recours à une source historique, Valère Maxime, afin de montrer que la chasteté de Scipion n'était pas si stricte qu'on le croit et que, lui aussi, comme César, a fait preuve de son «humanité »en commettant un péché de luxure. Jacopo Bracciolini situe le passage en question dans le cadre, plus vaste, de l'œuvre de Pétrarque, où Scipion est considéré comme le plus grand héros de l'Antiquité. Pour expliquer la place d'honneur accordée à César dans le Triomphe de la Renommée, il propose ensuite une interprétation philosophique du concept de renommée, concept qui doit être nettement distingué par rapport à celui de «vertu» et qui ne repose que sur la «voix du peuple».

Les trois commentateurs italiens abordent le texte sous des approches critiques complémentaires ; leur attention est focalisée sur l'opération littéraire de Pétrarque, sur les raisons et les fondements qui l'ont porté à placer César et Scipion sur un plan de parité. Leur attitude témoigne d'une conscience critique typiquement humaniste, proche de l'attitude moderne, où le texte est considéré moins comme l'instrument d'un message moral que comme l'objet d'une étude critique. Le commentateur français, par contre, est profondément ancré à une esthétique de type médiéval. Son travail d'exégèse se propose de mettre en exergue la valeur morale et exemplaire des deux personnages en question qui, par rapport à «l'amour de soi », c'est-à-dire au péché d'orgueil, incarnent deux modèles opposés, de vice (César) et de vertu (Scipion).

L'analyse de la figure de Jules César, des réactions qu'elle ne cesse pas de susciter jusqu'au seuil du XVI ${ }^{\mathrm{e}}$ siècle, nous a fourni l'occasion de proposer un aperçu de la riche tradition indirecte des Triomphes, tradition qui se situe à cheval entre Italie et France, entre texte et commentaire, entre Moyen Âge et Humanisme. 